\title{
How does Post-Mortem Imaging compare to autopsy, is this a relevant question?
}

Bruno Morgan ${ }^{1}$ PhD, FRCR, Guy N. Rutty ${ }^{2}$ MD, FRCPath

1. Imaging Department, University Hospitals of Leicester, Leicester Royal Infirmary, Leicester, LE1 5WW, United Kingdom. 2. East Midlands Forensic Pathology Unit, University of Leicester, Robert Kilpatrick Building, Leicester, LE2 7LX, United Kingdom

Corresponding author: Bruno Morgan, Imaging Department, University Hospitals of Leicester, Leicester Royal Infirmary, Leicester, LE1 5WW, United Kingdom

Tel: +44 1162523221 Fax: +44 1162523274

bm11@le.ac.uk

\section{Highlights}

Evidence of "efficacy" for a test does not imply it is effective in the real world Direct comparisons between autopsy and imaging may give misleading results Imaging is used in a wide variety of contexts, all with different questions The level of accuracy required of an imaging test depends on the context Research findings in one context may not be applicable in all contexts

\section{Abstract}

The rapid development of imaging techniques used for the investigation of death invites the question "how does imaging compare with autopsy". This article is based on an invited talk given to the International Society of Forensic Radiology and Imaging in 2015 and attempts to show that this question cannot be answered, as it is in fact several questions depending on the circumstances of death. Review of the literature is fraught with difficulties due to the rapid evolution of technology, the varied circumstances of death investigation and the multiple possible post-mortem investigations that imaging can be compared with. The article focuses on the assessment of adult sudden natural death and suggests the questions that need to be answered.

\section{Introduction}

Medical imaging is becoming increasingly common for the investigation of death. There is great debate about the relative qualities of imaging as compared with the traditional autopsy and many conclusions have already been made. However, this is not a straightforward debate and we believe it is complicated by a lack of comparative studies, vested interests, resistance to change [1] and probable misjudgements, based on misinterpretation of available data [2].

When a new imaging technique is developed it undergoes a series of testing (Health Technology Assessment, HTA), which can be summarised by the concepts of efficacy, effectiveness and efficiency. Efficacy and effectiveness are the extent to which a health technology influences a favourable outcome and are often used interchangeably, but are crucially different. Efficacy is a more technical term - does the test actually work, whereas effectiveness concerns the extent to which this 'efficacy' actually brings about the desired 
effect in the real world. For example a Magnetic Resonance Imaging (MRI) scan may be able to make a diagnosis accurately, but this has little impact or effectiveness if the diagnosis is untreatable or unimportant. An evaluative framework therefore must decide whether the test identifies the abnormalities, makes a diagnosis, displaces or improves upon other tests, contributes to service delivery and improves on outcomes for the whole population. Not surprisingly, most diagnostic tests have good evidence for their "efficacy" but often very little on their impact or effectiveness [3]. Focusing purely on technical performance can lead to failure to address the bigger picture and to incorrect assumptions about the new test. Therefore, before critically assessing the literature there must be a very clear concept of what overall effect or impact is required. Put simply, if we ask the wrong questions we will often get the wrong answer!

The purpose of this paper is to review what post-mortem imaging can do now and to try and frame the questions that need to be asked, both of previous studies and in new study design. However, after a review of the literature, the reader can come away with the impression that post-mortem (PM) imaging for the investigation of natural death is either very poor, giving a cause of death in less than $10 \%$ cases [4,5], or extremely good giving a cause of death in 90\% [6]. Similar discrepancies are also seen in unnatural death. All these papers are peer-reviewed and satisfy scientific scrutiny but they ask different questions, which have very different answers. This is the effect of having the same technical performance but with different measures as to the required effect.

We will show how by a combination of changing context, different biases, and inconsistent study comparators and end-points that it is very difficult to compare and even apply study findings to work in different areas. We will focus mainly on the investigation of adult sudden death, but also use information from other post-mortem investigation scenarios as examples.

\section{Context}

There are many reasons for a PM investigation, and these reasons dictate the questions to be asked. These include whether the death is of natural or unnatural cause, single or multiple, witnessed or un-witnessed, and also the age and identification of the dead. This opens up a vast range of possible scenarios where the key questions are very different, from medical cause of death, mechanism of death to victim identification. The purpose of the investigation will also vary depending on who has requested it. The police or the local coroner may have very specific questions that need to be asked, but investigation may also be requested for medical care reasons (the so-called hospital PM). This investigation is not only aimed to provide the principle cause of death, but also find all clinically relevant pathology to audit and educate clinical care.

It is also crucial to consider whether the medical imaging is being used as an adjunct to the standard PM investigation or a replacement. Use as an adjunct places less pressure on a test, as false negatives and false positives can be identified by and challenged by the traditional tests. Both tests together will normally have greater sensitivity $[7,8]$, and will therefore become the new gold standard. There is also growing interest in using medical imaging as a replacement for standard autopsy, both for natural and unnatural death. This is particularly true in England and Wales, due to a relatively high autopsy rate of $20 \%$ of natural deaths [9]. Although other countries do not have such high autopsy rates, there is also interest in providing PM imaging instead of autopsy internationally to increase the 
number of investigations (e.g. in Japan) and also to provide audit of medical care and education [10].

Another question is, "what competitor is imaging actually replacing"? This really should only be internal aspect of the traditional autopsy. Imaging cannot compete with the external examination of a body [11], and although this can be performed by imaging [12] it rarely needs to outside specific circumstances. Furthermore toxicology and even histology via targeted biopsy can be routinely added to an imaging examination $[13,14]$.

It is clear that when the context of the study changes then the prevalence of different diseases will also change. This pre-test probability will have a dramatic effect on the assessment of accuracy using the test. Many studies have different recruitment. For example, it is difficult to compare the results of excellent studies of hospital ITU deaths [7] compared with those from the community [15]. Likewise large studies and systematic reviews may have a very heterogeneous population, including both natural and unnatural deaths $[4,16]$. These statistical considerations are discussed in the next section.

\section{Bias in research methods and findings}

An on-line search reveals a multitude of potential biases that can affect the outcome of clinical trials. Autopsy and PM imaging studies are no different, with most studies showing "... marked variability in the methodology and the reporting quality, thus hindering ... the generalizability of the results [17]". Significant biases in research data are not always obvious and may be subtle. It is for this reason that a paper entitled "Why Most Published Research Findings Are False" has been heavily cited in the scientific literature and press [18].

It is natural at this point to call for more stringent peer-review and vetting prior to publication of scientific studies, but many scientists and editors no longer believe this to be either possible or appropriate. However well meaning, every form of review leads to a form of scientific censorship, and "claimed research findings may often be simply accurate measures of the prevailing bias [18]". Peer-review discourages publication of negative studies and also novel, small and eccentric studies. It often fails to identify falsehood and significant errors in experimental design and statistical analysis $[19,20]$. Furthermore, the electronic age is bringing greater capacity, with reducing costs for online only journals. Getting manuscripts accepted is therefore becoming easier and the ease of electronic searching and retrieval means that the brand-name of the journal is no longer crucial to disseminating information. This means that regardless of our view of peer-review, the reader should mistrust it and rely on our own "post-publication" peer-review, not just from 'letters to the editor' but on a comprehensive discussion of the "market of ideas" [20]. Ideally this would be supported by high quality systematic reviews, but these are only possible when there is enough data on a single circumstance of death with similar questions to study, for example traumatic death [21].

It is now therefore up to the individual to decide whether a study is scientifically accurate and also relevant to their own practice. A few examples are chosen based on the scientific content of the annual meeting of the International Society of Forensic Radiology and Imaging (ISFRI) 2015. None of the examples implies that the research method or the findings are wrong, simply that the reader should be vigilant.

Conference bias: There is no doubt that conferences benefit from submitted articles. Unlike many journals, the conference will often directly financially benefit from accepting a 
manuscript, by increased attendance. We served as the scientific review panel for (ISFRI) 2015 , and can vouch that all papers submitted were peer-reviewed. However, this was not a censorship process and nor was it strict quality control. It was a check to make sure submissions were intelligible, suitable for the meeting and for what session they were allocated to. To ascribe acceptance of a poster for the meeting as approval from the ISFRI itself would be incorrect.

Beware the invited review: When an expert is invited to review their field the peer-review process may not be particularly vigorous. To make sense of rapidly expanding knowledge most of us rely on these expert reviews to try to put some perspective on a subject and too much scrutiny will simply discourage experts from doing it. This does not imply that the review is false; simply that the experts' opinions and knowledge is often taken on trust. However, scientific reviews can propagate previous weak or even false findings so that they become part of perceived wisdom. Trying to find the origin of questionable dogma can sometimes be difficult and has been coined a "Woozle hunt" after A.A. Milne's book, Winnie the Pooh, where Pooh Bear and Piglet follow a Woozle's tracks, finding more and more tracks, until they realise they are only following themselves! Even when the "Woozle" is found, the problem often does not lie with the original story, but with the retelling. For example, we still hear people telling us that PM computed tomography (PMCT) is not very good for fractures, particularly those of the ribs and flat bones of the skull. We do not believe this as pathologists have to work hard at autopsy to even come close to detecting the amount of fractures seen on CT. In fact this is a good example of a simple question actually being quite difficult to answer, relying heavily on context, technique and experience.

Technical bias: If clinical CT scan reports from older CT scanners are compared with full dissection at autopsy, fractures are missed, particularly in the ribs and skull $[22,23]$. However, this does not necessarily compare to modern PMCT practice, which uses higher radiation-dose scans, thinner cuts and a different reporting approach [24]. For example, rib fractures normally hurt, and therefore "diagnose themselves" in life. These are therefore not routinely screened for in clinical practice, but are common to a forensic radiologist. Conversely, it is likely that a high quality PMCT scan, reported by a forensic radiologist, will identify more fractures than a rapid autopsy performed solely to establish the cause of death.

Coronary vascular imaging has been stated as a weakness of PM imaging $[17,25]$, but this is another area where the techniques of angiography in the dead [26-29] are changing the technical performance of imaging, which may even lead to greater effectiveness than standard autopsy techniques [30].

Pre-test probability: As already stated, different recruitment will change the prevalence of disease (ascertainment bias). This is particularly compounded with low recruitment numbers and one particular problem, typical in conference abstracts, occurs if a study with low numbers shows no adverse result or error. If no error is found in 10 cases this cannot be applied to the whole population and some idea of the confidence of the result is required. $A$ statistically sound but simple method for dealing with this is to assume that up to 3 measurements may be wrong (or adverse), the so-called rule of three [31]. This clearly has great impact if $n=10$ as 0 adverse events (or errors) becomes up to 3 potential adverse events or $30 \%$. If $n=1000$ there is more certainty as up to 3 adverse events is only $0.3 \%$. 
Simply assuming 3 data points are wrong in any series strikes us a safe way of interpreting any data-set.

Changing the question: PMCT can be insensitive for organ damage in trauma [32]. This can clearly be improved by using whole body angiography techniques [33]. However, it would be incorrect to assume that PMCT is not capable of assessing trauma. Indeed local experience shows us that organ trauma that has led to death is normally either visible or predictable. This is based on surrounding haemorrhage, and the absence of surrounding haemorrhage generally indicates that circulatory failure has occurred rapidly elsewhere. In this case the question "does PMCT identify organ lacerations" would have a different answer to "is PMCT sensitive to haemorrhage from significant organ lacerations"?

\section{What are the questions? How do we measure accuracy of the answer?}

Focussing on unexplained but not obviously unnatural death, the classic questions asked for the purposes of a Coroner's inquest in England and Wales are "who the deceased was and how, when and where the deceased came by his death? "How" can be extended to "by what means and in what circumstances", which gives a more some scope to factors leading to the death [34].

However, from a scientific point of view a more specific goal is required, so whether imaging can provide this, and with what accuracy, can be measured. The accuracy required will depend on the level of question.

1: Is the death really natural?

2: Can a cause of death be given to the standard required to satisfy the coroner's inquest?

3: Is the registered cause of death accurate for health-care planning?

4: Can the cause of death and other findings aid audit of medical care?

When reviewing different studies comparing imaging and autopsy, scoring systems to deal with discrepancies are often very different. Systems have been devised to assess accuracy of clinical diagnoses compared with autopsy $[35,36]$ and these have been adapted for use with PM imaging compared with autopsy [7]. However, these scoring systems and others used for imaging are often devised relating to technical performance or medical audit. For example a "major discrepancy" would be a failure to identify a diagnosis that could have changed medical management [36] or identifying the wrong organ system in the cause of death [15].

We would suggest that discrepancies are more appropriately analysed based on the above 4 questions for natural death. The accuracy and fitness-for-purpose of post-mortem investigation can then be assessed based on the level of certainty required for each question.

1: Is the death really natural? Failing to identify an unnatural death could miss a potentially criminal act - this therefore has to be answered to a level of "beyond reasonable doubt" the standard for a criminal court. Failure would be THE major discrepancy. For the same reasons victim identification is also crucial.

2: Does the result satisfy the Coroner? Once natural death is confirmed the "by what means" comes under the level of proof required for a coroner's court i.e. "on the balance of probabilities". Although it is tempting to assign "major discrepancy" to identifying the 
wrong organ system for a cause of death, for example bronchopneumonia vs. coronary heart disease, this has little impact on society and indeed the conditions may well co-exist. It is possible to come to a different set of "cause of death" conclusions, even when faced with the same data [37]. The "balance of probabilities" is not defined specifically, in fact neither is "beyond reasonable doubt", and so an acceptable " $p$ " number or confidence intervals cannot be stated. What is clear is that giving coronary vascular disease for all cases of adult sudden death would give the correct diagnosis more times than not [38] and would therefore be correct on the "balance of probabilities". This is clearly not an excuse for guessing, but it does raise the question of "how accurate must the test be". This will also relate to "how much does the test cost"? There is no doubt that PMCT, and particularly PMCTA with assessment of coronary arteries, can provide "a" cause of death "on the balance of probabilities" for most cases of adult natural sudden death. Logically it should also be cheaper than autopsy [39], although this is not currently the case in England and Wales [9].

3: Does it affect healthcare planning? Incorrect diagnoses will affect national death registration statistics. In England and Wales most registered deaths are not autopsied, and there is a considerable error rate in standard death certification [37]. Routine or medicolegal autopsies have also been shown to be inconsistent $[40,41]$. However, if a new system is initiated any systematic change in cause of death statistics should be recognised. For example, in some studies PMCT may fail to recognise pulmonary embolism as a cause of death [17], although this diagnosis is now being made [42] and is often given as a cause of death from PMCT in our centre. The same may also apply for autopsy; in the UK dementia is the most common cause of death in females over 80 years of age, but rarely features as a diagnosis post autopsy. An overall summary of the incidence of different diseases diagnosed by each test, which would show any potential systematic errors that may affect population data, will therefore be more informative than the number of individual discrepancies.

4: Audit of medical or institutional care: Multiple studies have shown that, even in hospital and intensive care environments, working diagnoses can be incorrect and subsequent postmortem investigation, including imaging, can provide useful learning points $[7,43,44]$.

\section{Conclusions}

When deciding whether a new imaging test is useful it is not just its technical performance (efficacy) that matters but whether it actually answers the questions required to a satisfactory standard (effectiveness) in different contexts and at what cost (efficiency). In this article, we argue that the ability of imaging to deliver post-mortem investigation is dependent on the circumstances of death. Without a clear idea of what effect is required from the post-mortem investigation it is impossible to design a study to measure the efficacy of the test. Simply measuring technical performance against findings at autopsy in a given scenario may not give a true measure of effectiveness and is also unlikely to translate to different circumstances of death.

The literature is growing but suffers from studies with a wide range of context, low recruitment numbers and lack of controls. Their applicability to practice in different countries and indications is therefore limited. However, the high quality studies that are required for drug development are problematic as there is less commercial interest, and grant funding often has to compete with medical studies for the living. The expansion of opportunities for publication and the perceived failure of peer-review will probably result in 
an increase in data that is potentially poorly presented and concluded. It is up to the scientific community to decide what information is useful and what is not, not necessarily to take the headline result but extract the useful information within. This review will be increasingly post-publication. This is considerably aided if systematic reviews are possible for specific circumstances of death, such as a recent review of traumatic death [21].

\section{Conflicts of interest}

None

\section{References}

1. Rutty J, Morgan B, Rutty GN. Managing transformational change: Implementing cross-sectional imaging into death investigation services in the United Kingdom. J Forensic Radiol Imaging. 2015;1:57-60.

2. Tversky A, Kahneman D. Judgment under uncertainty: Heuristics and biases. Science. 1974;185(4157):11241131.

3. Mackenzie R, Dixon AK. Measuring the effects of imaging: An evaluative framework. Clin Radiol. 1995;50(8):513-518.

4. Kasahara S, Makino Y, Hayakawa M, Yajima D, Ito H, Iwase H. Diagnosable and non-diagnosable causes of death by postmortem computed tomography: A review of 339 forensic cases. Leg Med (Tokyo). 2012;14(5):239245.

5. Bedford PJ. Routine CT scan combined with preliminary examination as a new method in determining the need for autopsy. Forensic Sci Med Pathol. 2012;8(4):390-394.

6. Bisset RA, Thomas NB, Turnbull IW, Lee S. Postmortem examinations using magnetic resonance imaging: Four year review of a working service. BMJ. 2002;324(7351):1423-1424.

7. Wichmann $D$, Obbelode $F$, Vogel $H$, et al. Virtual autopsy as an alternative to traditional medical autopsy in the intensive care unit: A prospective cohort study. Ann Intern Med. 2012;156(2):123-130.

8. Westphal SE, Apitzsch J, Penzkofer T, Mahnken AH, Knuchel R. Virtual CT autopsy in clinical pathology: Feasibility in clinical autopsies. Virchows Arch. 2012;461(2):211-219.

9. Rutty GN. Can cross-sectional imaging as an adjunct and/or alternative to the invasive autopsy be implemented within the NHS? Report from the NHS implementation sub-group of the department of health post mortem, forensic and disaster imaging group (PMFDI).

http://www2.le.ac.uk/departments/emfpu/national-documents Accessed July 2015.

10. Rutty GN, Morgan B. Virtual autopsy. Forensic Sci Med Pathol. 2013;9(3):433-434.

11. Rutty GN, Boyce $P$, Robinson CE, Jeffery AJ, Morgan B. The role of computed tomography in terminal ballistic analysis. Int J Legal Med. 2008;122(1):1-5.

12. Ebert LC, Ptacek W, Breitbeck R, et al. Virtobot 2.0: The future of automated surface documentation and CTguided needle placement in forensic medicine. Forensic Sci Med Pathol. 2014;10(2):179-186.

13. Bolliger SA, Filograna L, Spendlove D, Thali MJ, Dirnhofer S, Ross S. Postmortem imaging-guided biopsy as an adjuvant to minimally invasive autopsy with CT and postmortem angiography: A feasibility study. AJR Am J Roentgenol. 2010;195(5):1051-1056.

14. Rutty GN, Smith P, Visser T, Barber J, Amorosa J, Morgan B. The effect on toxicology, biochemistry and immunology investigations by the use of targeted post-mortem computed tomography angiography. Forensic Sci Int. 2013;225(1-3):42-47.

15. Roberts IS, Benamore RE, Benbow EW, et al. Post-mortem imaging as an alternative to autopsy in the diagnosis of adult deaths: A validation study. Lancet. 2012;379(9811):136-142.

16. Thayyil S, Chandrasekaran M, Chitty LS, et al. Diagnostic accuracy of post-mortem magnetic resonance imaging in fetuses, children and adults: A systematic review. Eur J Radiol. 2010;75(1):e142-8.

17. Burton EC, Mossa-Basha M. To image or to autopsy? Ann Intern Med. 2012;156(2):158-159.

18. loannidis JPA. Why most published research findings are false. PLoS Med. 2005;2(8):e124.

19. Smith R. Peer review: A flawed process at the heart of science and journals. J R Soc Med. 2006;99(4):178182.

20. Smith R. Screening for cardiovascular disease using age alone: Reflections on a paper peer-reviewed as both 'radical' and 'unsurprising'. J Med Screen. 2011;18(3):113-114.

21. Jalalzadeh H, Giannakopoulos GF, Berger FH, et al. Post-mortem imaging compared with autopsy in trauma victims - A systematic review. Forensic Sci Int. 2015;257:29-48. 
22. Molina DK, Nichols JJ, Dimaio VJ. The sensitivity of computed tomography (CT) scans in detecting trauma: Are CT scans reliable enough for courtroom testimony? J Trauma. 2007;63(3):625-629.

23. Jacobsen $C$, Bech BH, Lynnerup N. A comparative study of cranial, blunt trauma fractures as seen at medicolegal autopsy and by computed tomography. BMC Med Imaging. 2009;9:18-2342-9-18.

24. Rutty GN, Morgan B, O'Donnell C, Leth PM, Thali M. Forensic institutes across the world place CT or MRI scanners or both into their mortuaries. J Trauma. 2008;65(2):493-494.

25. Roberts IS, Benbow EW, Bisset R, et al. Accuracy of magnetic resonance imaging in determining cause of sudden death in adults: Comparison with conventional autopsy. Histopathology. 2003;42(5):424-430.

26. Ross SG, Thali MJ, Bolliger S, Germerott T, Ruder TD, Flach PM. Sudden death after chest pain: Feasibility of virtual autopsy with postmortem CT angiography and biopsy. Radiology. 2012;264(1):250-259.

27. Saunders SL, Morgan B, Raj V, Robinson CE, Rutty GN. Targeted post-mortem computed tomography cardiac angiography: Proof of concept. Int J Legal Med. 2011;125(4):609-616.

28. Roberts IS, Benamore RE, Peebles C, Roobottom C, Traill ZC. Technical report: Diagnosis of coronary artery disease using minimally invasive autopsy: Evaluation of a novel method of post-mortem coronary CT angiography. Clin Radiol. 2011;66(7):645-650.

29. Morgan B, Sakamoto N, Shiotani S, Grabherr S. Postmortem computed tomography (PMCT) scanning with angiography (PMCTA): A description of three distinct methods. In: Rutty GN, ed. Essential of autopsy practice. Advances, updates and emerging technologies. Springer, London; 2014:1-22.

30. Morgan B, Biggs MJ, Barber J, et al. Accuracy of targeted post-mortem computed tomography coronary angiography compared to assessment of serial histological sections. Int J Legal Med. 2013;127(4):809-817. 31. Eypasch E, Lefering R, Kum CK, Troidl H. Probability of adverse events that have not yet occurred: $A$ statistical reminder. BMJ. 1995;311(7005):619-620.

32. Christe A, Ross $S$, Oesterhelweg L, et al. Abdominal trauma--sensitivity and specificity of postmortem noncontrast imaging findings compared with autopsy findings. J Trauma. 2009;66(5):1302-1307.

33. Grabherr S, Dominguez A, Mangin P. Post-mortem angio-CT: A new diagnostic approach. Rev Med Suisse. 2011;7(303):1507-1510.

34. Dorries C. Coroners' courts: A guide to law and practice. 2nd edition ed. Oxford: Oxford University Press; 2004.

35. Goldman L, Sayson R, Robbins S, Cohn LH, Bettmann M, Weisberg M. The value of the autopsy in three medical eras. N Engl J Med. 1983;308(17):1000-1005.

36. Dimopoulos G, Piagnerelli M, Berre J, Salmon I, Vincent JL. Post mortem examination in the intensive care unit: Still useful? Intensive Care Med. 2004;30(11):2080-2085.

37. Pritt BS, Hardin NJ, Richmond JA, Shapiro SL. Death certification errors at an academic institution. Arch Pathol Lab Med. 2005;129(11):1476-1479.

38. Biggs MJ, Brown LJ, Rutty GN. Can cause of death be predicted from the pre-necropsy information provided in coroners' cases? J Clin Pathol. 2008;61(1):124-126.

39. Weustink AC, Hunink MG, van Dijke CF, Renken NS, Krestin GP, Oosterhuis JW. Minimally invasive autopsy: An alternative to conventional autopsy? Radiology. 2009;250(3):897-904.

40. Lucas SB, Cooper H, Emmett S, Hargraves C, Mason M. The coroner's Autopsy: Do we deserve better? A report of the national confidential enquiry into patient outcome and death.

www.ncepod.org.uk/2006Report/Downloads/summary.pdf Last accessed July 2015

41. Luce T. Coroners and death certification law reform: The coroners and justice act 2009 and its aftermath. Med Sci Law. 2010;50(4):171-178.

42. Ruder TD, Thali Y, Hatch GM, Thali MJ, Ampanozi G. Evaluation of vascular diameter and cases of pulmonary embolism sudden death. Journal of Forensic Radiology and Imaging. 2014;2(2):100.

43. Shojania KG, Burton EC, McDonald KM, Goldman L. Changes in rates of autopsy-detected diagnostic errors over time: A systematic review. JAMA. 2003;289(21):2849-2856.

44. Nichols L, Aronica P, Babe C. Are autopsies obsolete? Am J Clin Pathol. 1998;110(2):210-218. 